\title{
Biodisponibilité de quelques métaux fixés sur les matières en suspension (MES) en milieu estuarien
}

\author{
H. Ettajani et J.C. Amiard(1) \\ (1) Service d'Ecotoxicologie, EP 61 du CNRS, Université de Nantes, 1, rue Gaston Veil, 44035 \\ Nantes Cedex (France).
}

\begin{abstract}
Résumé. - Dans les milieux aquatiques, un grand nombre de polluants, surtout les métaux, se fixent sur les matières en suspension (MES). Ingérées par les organismes benthiques, ces MES subiront l'ensemble du processus de digestion (incluant les variations $\mathrm{du} \mathrm{pH}$, et l'action des enzymes digestives). Une partie des métaux adsorbés aux particules sera biodisponible entraînant une augmentation de la concentration de ces éléments dans l'animal.

Dans le but d'évaluer cette biodisponibilité, une étude expérimentale a été réalisée avec du seston naturel prélevé au printemps et en été au niveau du bouchon vaseux de l'estuaire de la Loire. Ce seston a été soumis à différents traitements: changements de la salinité et du $\mathrm{pH}(0,1 \mathrm{~N} \mathrm{HCl}, \mathrm{pH}$ rencontrés dans le tube digestif des Bivalves) et des principales enzymes digestives des mollusques filtreurs.

Les résultats montrent que, selon les saisons, la désorption des métaux est différente. En effet, sous l'action de la salinité, la mobilité des métaux pour le prélévement estival n'est mise en évidence que pour l'As et le $\mathrm{Pb}$, alors que pour celui du printemps, tous les métaux sont concernés ( $\mathrm{Ag} . \mathrm{As}, \mathrm{Cd}, \mathrm{Cu}, \mathrm{Pb}, \mathrm{Zn}$ ). II en est de même pour les attaques enzymatiques, les enzymes digestives ayant une action différente sur le relargage du $\mathrm{Cd}$ au printemps et en été. L'effet d'une attaque acide relativement modérée ( $\mathrm{HCl} \mathrm{0,1} \mathrm{N}$ ) entraine des désorptions plus importantes au printemps qu'en été.
\end{abstract}

Mots clés. - Attaques enzymatiques, Matières en suspension, Biodisponibilité, Métaux (Ag, As, Cd, Cu, $\mathrm{Pb}, \mathrm{Zn}$ ), Salinité, Estuaire.

Abstract. - In the aquatic environment, a great number of pollutants, especially heavy metals, are adsorbed to suspended matter. Once ingested by benthic organisms, particles are submitted to digestion processes (including $\mathrm{pH}$ changes and the action of digestive enzymes). A part of particle-bound metals may be bioavailable leading to an enhancement of metal concentrations in organisms.

In order to evaluate this bioavailability, an experimental study has been carried out using naturel sediment sampled at spring and summer from the Loire estuary. This sediment was submitted to different treatments : salinity and $\mathrm{pHs}$ changes $10.1 \mathrm{~N} \mathrm{HCl}, \mathrm{pHs}$ en- 
countered in the digestive tract of bivalves) and the action of the main digestive enzymes of filter-feeders.

Results showed that metal desorption differs depending on the seasons. Thus, metal mobility due to salinity variation was evidenced only for $\mathrm{As}$ and $\mathrm{Pb}$ in the case of summer sediment, whereas for spring sediment, all the metals were concerned $(\mathrm{Ag}, \mathrm{As}, \mathrm{Cd}, \mathrm{Cu}$, $\mathrm{Pb}, \mathrm{Zn}$ ). For enzyme attacks also, Cd desorption due to digestive enzymes was different in spring and summer. Weakly-concentrated acid $(0.1 \mathrm{~N} \mathrm{HCl})$ action induced more important metal release from spring than from summer sediment.

Key words. - Enzyme attack, Suspended matter, Bioavailability, Metals (Ag, As, Cd, $\mathrm{Cu}, \mathrm{Pb}, \mathrm{Zn}$ ) Salinity, Estuary.

\section{INTRODUCTION}

Dans l'environnement aquatique, un grand nombre de polluants se fixent sur les particules sédimentaires, qu'elles soient déposées ou en suspension. Ce phénomène se déroule aussi bien en milieux dulçaquicole que saumâtre et marin.

Les flux de polluants entre les divers compartiments du milieu aquatique varient en fonction de la spéciation chimique des polluants qui dépend à la fois de la nature de la substance chimique introduite dans le milieu et des caractéristiques physicochimiques de ce dernier. Dans les phases aqueuse et particulaire, la mobilité de la substance sera dépendante de facteurs chimiques, biologiques et physiques (Förstner, 1990). Au niveau des particules sédimentaires, les formes physico-chimiques de liaison expliquent en grande partie les échanges entre phases solide et liquide.

Cependant, la biodisponibilité d'une substance liée au sédiment ne peut se résumer à sa capacité à se solubiliser dans la phase aqueuse sous l'action de facteurs externes. En effet, les organismes benthiques se nourrissent en ingérant des particules sédimentaires riches en matière organique et éventuellement en polluants. Au cours du transit digestif, l'aliment subit des transformations chimiques avec notamment des variations du $\mathrm{pH}$. Ces attaques chimiques peuvent libérer dans la lumière du tube digestif les substances préalablement liées aux particules sédimentaires. Une fois libre dans le tube digestif, la substance pourra pénétrer dans l'organisme sous forme ionique ou liée à une molécule biologique ayant un rôle de transporteur transmembranaire. Une substance polluante peut donc être solubilisée dans la phase aqueuse ou dans le tube digestif des organismes benthiques. Cette dernière voie a été très négligée et peu d'études ont essayé d'identifier son importance.

Sous nos latitudes la plupart des estuaires sont soumis aux marées et la présence d'un bouchon vaseux a été mise en évidence. Un grand nombre de polluants, et particulièrement certains métaux ou métalloïdes, se fixent fortement sur les particules, 
inertes ou vivantes, organiques ou inorganiques, constituant ce bouchon vaseux. Les quantités expulsées seront fonction du régime fluvial, étiage ou crue, et du coefficient de marée (vives ou mortes eaux). Avant 1976, le pourcentage de matériaux expulsé hors de l'estuaire de la Loire est de 50 à $60 \%$ tandis qu'après 1976 , ce pourcentage n'est plus que de $30 \%$; ce phénomène serait dû aux dragages réalisés au niveau du chenal d'entrée de l'estuaire (CSEEL, 1984). Lors de leur expulsion, les particules sédimentaires passent par un gradient de salinité depuis l'eau douce jusqu'à une salinité voisine de celle de l'eau de mer $(35 \% \circ)$. La modification de la salinité, et donc de la force ionique du milieu, aura une répercussion sur le pouvoir de fixation des ions métalliques. Selon les périodes et seIon les auteurs, les estuaires ont été considérés comme des puits ou comme des sources pour les métaux vis-à-vis du milieu matin.

En arrivant dans l'estuaire externe de la Loire et sur le proche littoral, les particules sédimentaires riches en métaux, seront absorbées par les organismes benthiques en même temps que leur nourriture. Ces particules sont très diverses tant par leur nature que par leur taille et seule une fraction sera sélectionnée par les Etres Vivants et subira le processus de la digestion. Une partie des métaux liés à ces particules sera biodisponible pour les organismes benthiques, entraînant une augmentation de la concentration de ces éléments dans l'animal. Or, parmi les organismes benthiques, certains servent fréquemment d'aliment à l'Homme comme quelques Mollusques intrasédimentaires et/ou filtreurs (Huitre, Moule, Coque, Scrobiculaire).

Nous nous proposons de quantifier la biodisponibilité de divers métaux ( $\mathrm{Ag}, \mathrm{As}, \mathrm{Cd}, \mathrm{Cu}, \mathrm{Pb}$ et $\mathrm{Zn}$ ) fixés sur le bouchon vaseux de l'estuaire de la Loire en fonction de la saison et de la nature des particules.

\section{MATÉRIEL ET MÉTHODES}

\subsection{Prélèvements et caractéristi- ques des échantillons}

Les prélèvements du bouchon vaseux de l'estuaire de la Loire ont été effectués à deux saisons, au printemps (20/03/1992) et en été (31/07/1992). Chaque prélèvement est effectué en surface et à $1 \mathrm{~m}$ du fond, c'est-à-dire dans la crème de vase. Les caractéristiques du milieu lors des prélèvements sont résumées dans le tableau I. Les mesures ont été effectuées par les équipes du Port Autonome Nantes Saint-Nazaire à l'aide du système SYMEL (Système de Mesure sur l'Estuaire de la Loire).

Les sédiments sont ramenés au laboratoire à l'abri de l'air. Ils sont centrifugés et congelés à $-20^{\circ} \mathrm{C}$. Les principales caractéristiques des sédi- 
Tableau I. - Caractéristiques du milieu à proximité du bouchon vaseux lors des deux périodes de prélèvements.

\begin{tabular}{|c|c|c|c|c|}
\hline Prélèvements & $\begin{array}{cc} & 20 / 03 / 92 \\
& \text { surface }\end{array}$ & $\begin{array}{l}20 / 03 / 92 \\
\text { fond }\end{array}$ & $\begin{array}{c}31 / 07 / 92 \\
\text { surface }\end{array}$ & $\begin{array}{c}31 / 07 / 92 \\
\text { fond }\end{array}$ \\
\hline Conductivité $(\mu \mathrm{S} / \mathrm{cm})$ & 481 & 538 & 25400 & 19200 \\
\hline Température $\left({ }^{\circ} \mathrm{C}\right)$ & 11,1 & 11,4 & 17.9 & 17,3 \\
\hline $\mathrm{O}_{2}$ dissous $(\mathrm{mg} / \mathrm{l})$ & 6,9 & 1,1 & 2.5 & 0,9 \\
\hline & 8 & 8,1 & 7,01 & 7,32 \\
\hline Salinité (\%) & 0,34 & 0,38 & 21.6 & 16,32 \\
\hline
\end{tabular}

\section{Tableau II}

A. - Caractéristiques des sédiments du bouchon vaseux aux deux périodes de prélèvements.

B. - Concentrations métalliques $(\mu \mathrm{g} / \mathrm{g} \mathrm{sec})$ du sédiment de surface (S) et de fond (F) du bouchon vaseux prélevé au printemps et en été.

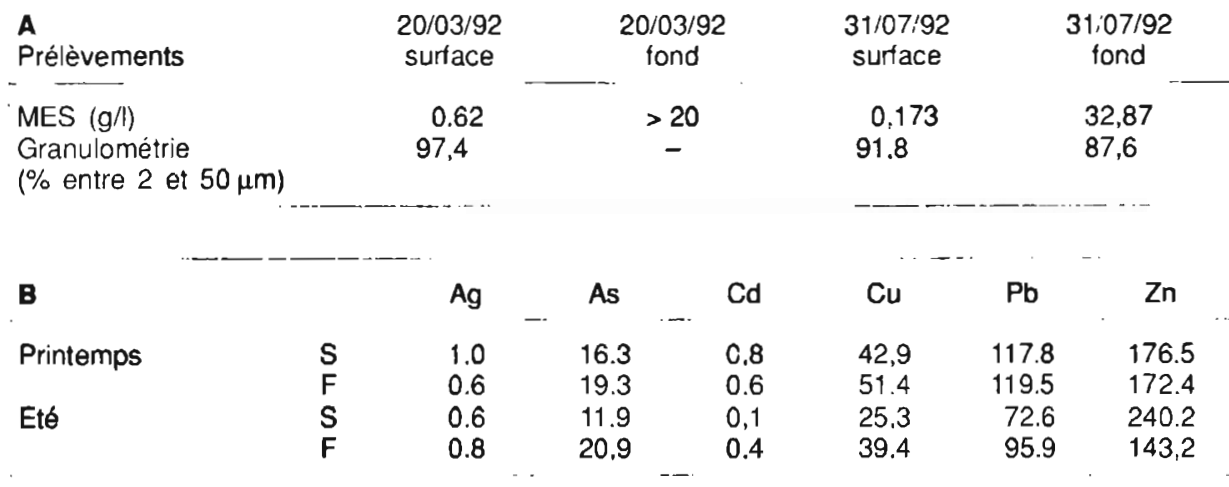

ments sont rapportées dans les tableaux II A et II B.

\subsection{Quantification de la désorption des métaux liés à la matiere en suspension}

\subsubsection{Influence de la salinité}

Une charge d'environ $50 \mathrm{mg}$ de sédiment est introduite dans $50 \mathrm{ml}$ deau de mer artificielle (salinité $33 \%$, $\mathrm{pH} 8,2 \mathrm{j}$. Le sédiment est agité pendant $24 \mathrm{~h}$ puis centrifugé $(5000 \mathrm{t} / \mathrm{min}$,
$15 \mathrm{~min}$ ). Le culot est minéralisé avec de l'acide nitrique (Suprapur) en vue du dosage des éléments traces.

\subsubsection{Attaque du sédiment par un} acide faiblement concentré $\mathrm{HCl} 0,1 \mathrm{~N}$

Une charge particulaire d'environ $50 \mathrm{mg}$ est attaquée par $2 \mathrm{ml}$ dacide chlorhydrique $0,1 \mathrm{~N}(\mathrm{HCl}$ Merck $30 \%$ ) durant $6 \mathrm{~h}$. Le sédiment est agité pendant ce laps de temps purs centrifugé. Le culot est minéraiısé avec de l'acide 
nitrique en vue du dosage des éléments traces.

\subsubsection{Simulations de la digestion d'un Mollusque filtreur}

D'après des études de dynamique et d'enzymologie de la digestion chez les Mollusques (Boucaud-Camou et al., 1983; Lebesnerais, 1985; Deslous-Paoli, 1987), le choix des principales enzymes présentes dans le tube digestif de l'Huître a porté sur: l' $\alpha$-amylase, la pepsine, la lysosyme, la peptidase et les phosphatases acide et alcaline. Des enzymes équivalentes proposées par divers fournisseurs ont été utilisées: $\alpha$-amylase (pancréas de porc), lysosome (muramidase) de chez Merck-Clévenot; phosphatase acide (Bovine prostate), phosphatase alcaline Type XVI (Porcine), pepsine (Muqueuse stomacale de porc), peptidase (Muqueuse intestinale de porc) de chez Sigma. Chaque enzyme a été mise en contact avec le sédiment en présence d'un tampon permettant de recréer le $\mathrm{pH}$ auquel fonctionne normalement l'enzyme : $\mathrm{pH} 4$ pour la pepsine, $\mathrm{pH} 5,5$ pour la lysosyme, la peptidase et la phosphatase acide et $\mathrm{pH} 6,8$ pour la phosphatase alcaline et l' $\alpha$-amylase.

Le pH (4, 5,5 et 6,8 en fonction des enzymes étudiées) est obtenu avec un tampon acide acétique 1\% (Normapur). L'action du tampon à différents $\mathrm{pH}$ a été testée concomitamment aux essais de désorption des métaux par les enzymes. Les attaques enzymatiques ont été réalisées à température ambiante pendant $6 \mathrm{~h}$ correspondant à la durée moyenne de séjour des aliments dans le tube digestif de l'huittre (Lebesnerais, 1985). Pour chaque unité expérimentale, la quantité d'enzyme introduite est de $1 \mathrm{mg}$ pour $2 \mathrm{ml}$ de tampon et $50 \mathrm{mg}$ de sédiment. A la fin de l'expérience le sédiment est centrifugé ( $5000 \mathrm{t} / \mathrm{min}$ pendant $10 \mathrm{~min}$ ), rincé avec le tampon acide acétique $(\mathrm{pH} 4$, $5,5$ ou 6,8$)$, de nouveau centrifugé. Le culot est minéralisé avec de l'acide nitrique. Selon les prélèvements (sédiment de fond ou de surface), les expériences sont renouvelées deux ou trois fois pour les divers traitements.

\subsection{Analyses globales par spec- trophotométrie d'absorption ato- mique}

Les particules sédimentaires à analyser, de poids sec connu, sont attaquées par de l'acide nitrique suprapur à chaud $\left(70^{\circ} \mathrm{C}\right)$. Le résidu non digéré est éliminé et le volume de dissolution est complété à $4 \mathrm{ml}$ avec de l'eau déminéralisée. Le dosage des métaux dans ces solutions est ensuite effectué par spectrophotométrie d'absorption atomique de flamme ou électrothermique (Hitachi Z8100 ou PerkinElmer 3030) selon les procédures décrites par Amiard et al. (1987).

\section{RÉSULTATS ET DISCUSSION}

\subsection{Concentrations globales des métaux dans les particules sédi- mentaires}

Les concentrations métalliques dans les particules sédimentaires sont présentées dans le tableau IIB. Les 
concentrations métalliques initiales au niveau du sédiment (de surface ou de fond) prélevé au printemps sont relativement supérieures à celles du sédiment estival. Cette différence est relativement plus importante pour $\mathrm{Cd}$, $\mathrm{Cu}$ et $\mathrm{Pb}$. Ceci pourrait être dû à un effet de la salinité. En effet, en été les particules prélevées sont en contact avec une eau dont la salinité est plus élevée $(16,32$ et $21,6 \%$, respectivement pour les particules de fond et de surface) qu'au printemps $(0,34$ et $0,38 \% \circ)$. De même, le débit moyen de la Loire est plus faible en été qu'au printemps amenant un séjour prolongé des particules dans la zone d'accumulation turbide (bouchon vaseux). Cet allongement du temps de contact permettrait selon El Sayed (1988) aux processus de désorption et de dégradation de la matière organique d'accentuer leur effet et de libérer le cuivre particulaire. C'est ainsi que dans l'estuaire de la Loire, selon Seyler (1985), les concentrations en arsenic particulaire diminuent d'amont en aval avec toutefois une augmentation entre Nantes et Cordemais due à la pollution d'origine humaine. De plus, Boust (1981) a montré qu'en étiage dans l'estuaire de la Seine le rôle de la salinité se fait nettement sentir par la modification du cortège des éléments extractibles rendue possible par le temps de résidence plus long des particules dans l'estuaire.

\subsection{Influence de la salinité}

L'effet de la salinité (33\%o) sur la désorption des éléments fixés aux particules du bouchon vaseux est net- tement plus important sur les particules prélevées au printemps qu'en été et ceci aussi bien pour le sédiment de surface que pour la crème de vase (Fig. 1, A et B). Au printemps l'ordre de désorption des métaux liés à la crème de vase est $\mathrm{Zn}>\mathrm{As}>\mathrm{Cu}>\mathrm{Pb}>\mathrm{Ag}>\mathrm{Cd}$ et pour le sédiment de surface $\mathrm{Ag}>\mathrm{As}-$ $\mathrm{Cu}>\mathrm{Zn}>\mathrm{Pb}>\mathrm{Cd}$. En été, seuls As et $\mathrm{Pb}$ sont légèrement désorbés de la crème de vase pour des salinités de $33 \%$ (respectivement pour les particules de fond et de surface 17,2 et $23,2 \%$ pour As et 3,3 et $17 \%$ pour $\mathrm{Pb})$. Cette différence de comportement des métaux vis à vis du changement de salinité a déjà été relevé par Maest et al. (1990) pour l'estuaire du Raritan (New Jersey, USA). Ces auteurs ont constaté que les métaux pouvaient se répartir en trois groupes en fonction de leur comportement vis à vis des changements de salinité : le premier groupe ( $\mathrm{Fe}, \mathrm{Al}$ et $\mathrm{Cr}$ ) où la concentration dans la phase dissoute présentait une brusque augmentation à $4 \%$ puis revenait à un niveau faible et relativement stable; le deuxième groupe ( $\mathrm{Mn}, \mathrm{Cu}, \mathrm{Pb}$, Co et $\mathrm{Cd}$ ) présentait également une augmentation très nette de la concentration dans la phase dissoute à $4 \%$, des variations aléatoires laissant supposer l'intervention de sources de pollution; le troisième groupe ( $\mathrm{Ni}$ et $\mathrm{Zn}$ ) où l'augmentation de la concentration dans la phase dissoute est régulière et commence dès $4 \%$ pour $\mathrm{Ni}$ et seulement à $11,5 \%$ pour $\mathrm{Zn}$.

Dans l'estuaire du Saint-Laurent les concentrations de $\mathrm{Fe}, \mathrm{Mn}$ et $\mathrm{Zn}$ 

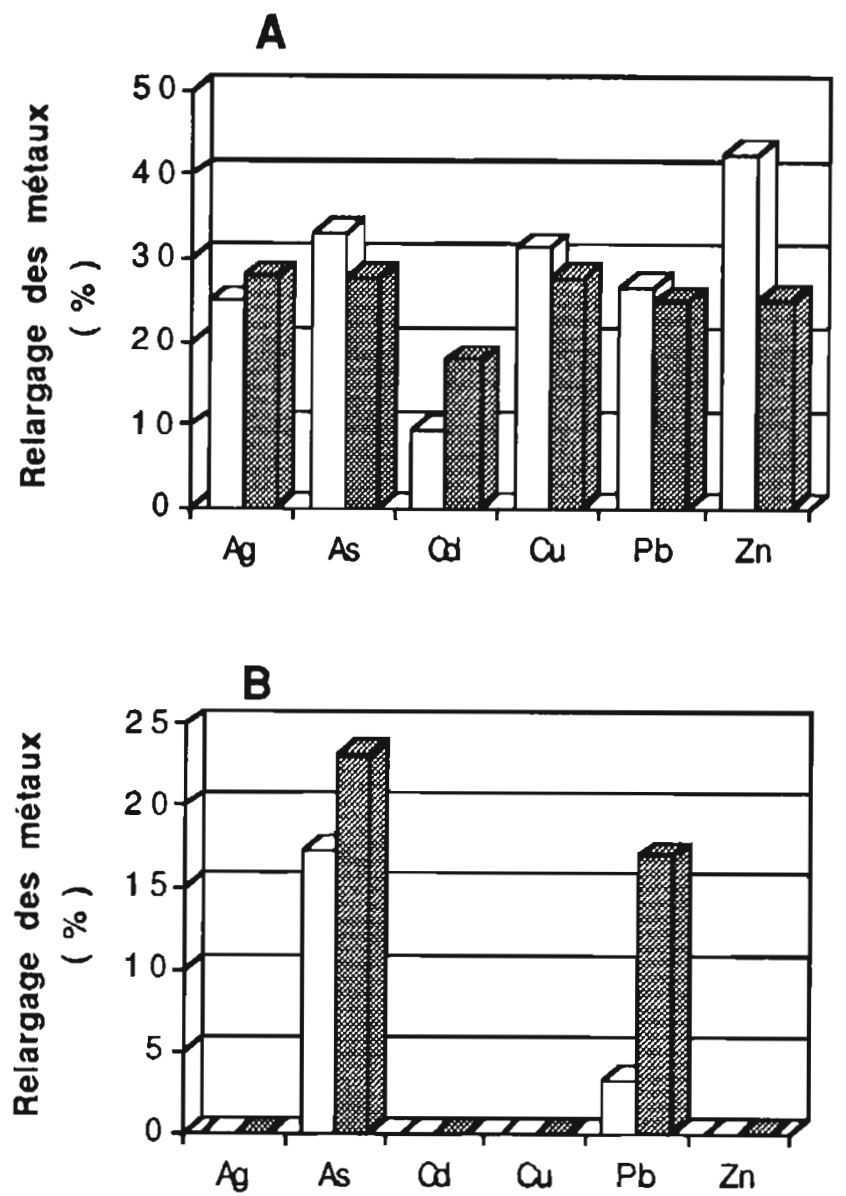

Fig. 1. - Pourcentages de désorption des éléments traces liés aux particules du bouchon vaseux de l'estuaire de la Loire par effet de la salinite $(33 \%)$ lors de prélèvements printaniers (A) et estivaux (B). Les aires blanches correspondent aux prélèvements de fond (crème de vase) et les aires hachurées aux prélèvements de surface.

fixés sur les matières en suspension détritiques diminuent quand la salinité augmente; le phénomène est inverse pour $\mathrm{Al}, \mathrm{Fe}, \mathrm{Mn}, \mathrm{Zn}$ et $\mathrm{Cu}$ liés aux matières en suspension non détritiques (Yeats et Loring, 1991). Dans l'estuaire de la Gironde, les concentrations de $\mathrm{Zn}, \mathrm{Ni}$, $\mathrm{Cu}$ et $\mathrm{Pb}$ liées aux particules diminuent lorsque la salinité augmente et seci se vérifie surtout en période de crue où les particules perdent 70 à $80 \%$ du zinc lié (Etcheber 1979 ; Latouche et al., 1982 ; Jouanneau et al., 1983). Par contre, dans le haut estuaire, la décroissance des concentrations métalliques n'est pas 
due à la salinité mais à un appauvrissement en carbone organique particulaire (COP) et en métaux dû à un mélange des particules estuariennes avec des particules marines en période de crue et à la remise en suspension en période d'étiage (Jouanneau et al., 1983). Nirel (1987) étudiant l'évolution de la distribution particulaire des éléments chimiques en milieu estuarien constate des différences selon les éléments et selon les estuaires. Ainsi pour la Gironde, les concentrations métalliques diminuent en fonction de la salinité pour $\mathrm{As}, \mathrm{Au}, \mathrm{Ba}, \mathrm{Ca}, \mathrm{Ce}, \mathrm{Cr}, \mathrm{Hf}, \mathrm{Sb}$ et $\mathrm{Zn}$, augmentent pour $\mathrm{Na}, \mathrm{Br}$ et $\mathrm{Ag}$ et restent stables pour $\mathrm{Rb}, \mathrm{Cs}$ et les terres rares. Pour l'estuaire de la Loire, les concentrations métalliques diminuent en fonction de la salinité pour $\mathrm{U}, \mathrm{Cs}$, $\mathrm{Ba}, \mathrm{Ca}, \mathrm{Se}, \mathrm{Zr}$, Sb et $\mathrm{Zn}$, augmentent pour $\mathrm{Na}$, Br et $\mathrm{Cr}$ - avec pour cet élément des sources de pollution manifeste dans cet estuaire - et restent stables pour $\mathrm{Co}, \mathrm{Fe}, \mathrm{Hf}, \mathrm{Rb}, \mathrm{Sc}, \mathrm{Ta}$, $\mathrm{Th}, \mathrm{Yb}$ et les terres rares. Les concentrations d'arsenic particulaire diminuent quand la salinité augmente.

\subsection{Attaque du sédiment par un acide faiblement concentré $\mathrm{HCl} 0,1 \mathrm{~N}$}

L'effet d'une attaque acide relativement modérée $(\mathrm{HCl} 0,1 \mathrm{~N})$ entraîne des désorptions plus importantes au printemps qu'en été surtout pour $\mathrm{Ag}$, As et $\mathrm{Cu}$ (fig. 2, $\mathrm{A}$ et B). Au printemps l'ordre de désorption est le suivant $\mathrm{Cd}>\mathrm{Pb}>\mathrm{Cu}=\mathrm{Zn}>\mathrm{Ag}>\mathrm{As}$. En été. l'ordre de désorption est identique mais $\mathrm{Ag}$ et As ne sont nullement désorbés de la crème de vase. Dans le cas des particules de surface, aussi bien pour le printemps que pour l'été, le pourcentage de cadmium relargué est nul, alors que pour les autres métaux il n'est pas négligeable $(\mathrm{Pb}=\mathrm{Zn}>\mathrm{Cu}>\mathrm{As}>\mathrm{Ag})$.

Plusieurs auteurs ont essayé d'utiliser l'attaque par l'acide chlorhydrique dilué pour estimer la biodisponibilité (Bryan, 1984 ; Campbell et al., 1988). Chez Nereis diversicolor par exemple, il a été montré que les niveaux d'argent, de cadmium, de cobalt et de zinc étaient mieux corrélés avec les concentrations dans certains extraits (acétate d'ammonium $1 \mathrm{~N}$ pour $\mathrm{Zn}$, $\mathrm{HCl} 1 \mathrm{~N}$ pour les autres métaux) qu'avec les concentrations globales dans le sédiment. Des résultats similaires ont été obtenus pour l'accumulation du $\mathrm{Cu}$, du $\mathrm{Zn}$ et du $\mathrm{Pb}$ chez des Pélécypodes d'eau douce (Tessier et al., 1984). Par contre, la disponibilité du plomb est aussi bien corrélée aux concentrations totales dans le sédiment qu'aux concentrations extractibles (Luoma, 1983). Ces distorsions s'expliquent dans la mesure où ces extractions ne peuvent pas mimer les systèmes biologiques mis en jeu dans les processus de résorption et d'extraction des éléments traces adsorbés aux niveaux des particules. Une interprétation plus poussée des extractions séquentielles en terme de biodisponibilité nécessite une méthodologie qui intègre les connaissances sur les conditions physico-chimiques subies par les particules au cours de la digestion. 

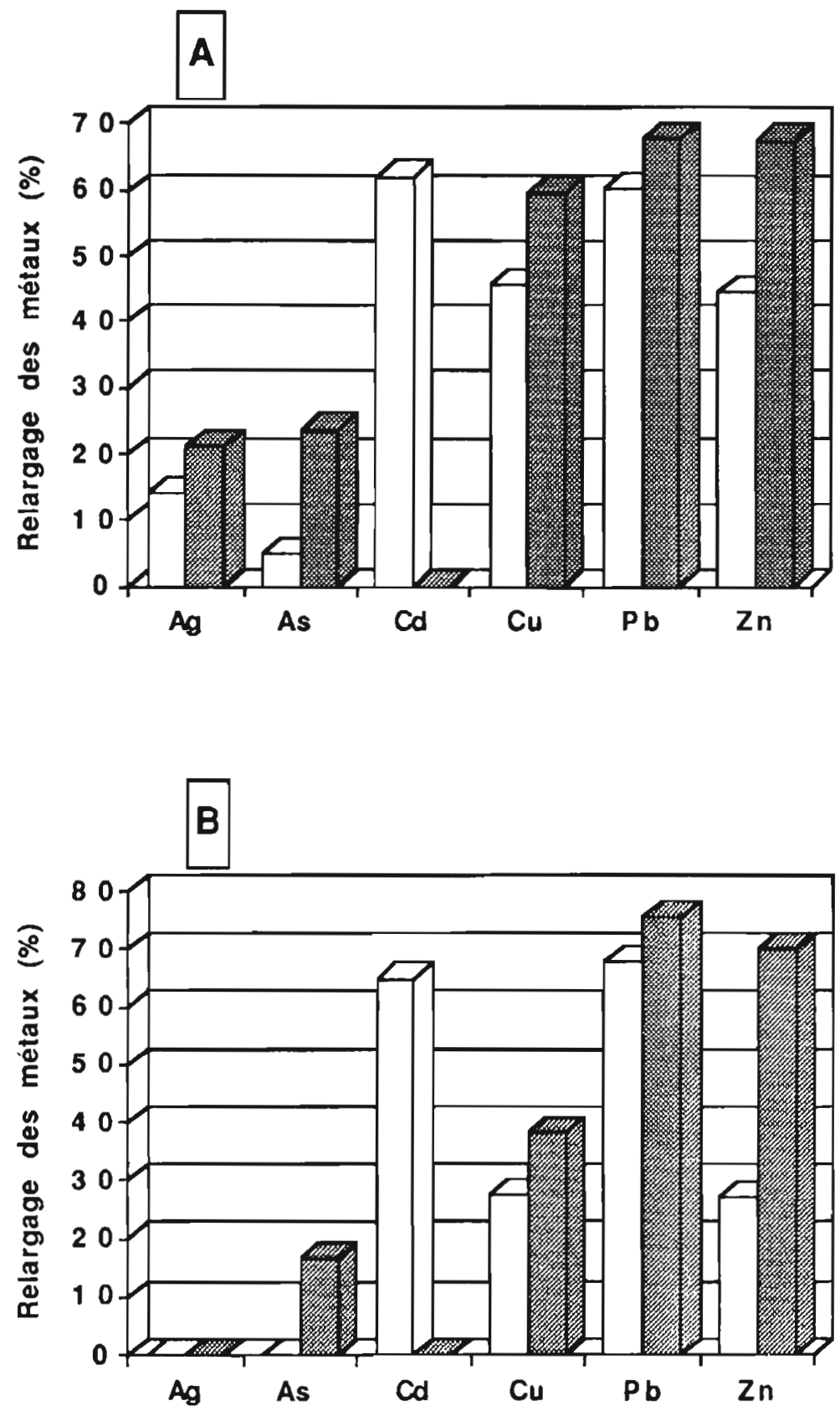

Fig. 2. - Pourcentages de désorption des éléments traces liés aux particules du bouchon vaseux de l'estuaire de la Loire par attaque acide $(\mathrm{HCl} 0,1 \mathrm{~N})$ lors de prélevements printaniers $(\mathrm{A})$ et estivaux (B). Les aires blanches correspondent aux prélèvements de fond (crème de vase) et les aires hachurées aux prélèvements de surface. 
L'influence du pH sur la mobilité ou simulant la biodisponibilité des métaux a été estimé par Boniforti et al. (1988) pour divers sédiments à l'aide de quatre traitements : $\mathrm{pH} 3$ simulant la digestion des Poissons, $\mathrm{pH} 6$ simulant la digestion des Invertébrés, $\mathrm{pH} 1-1,2$ avec $0,5 \mathrm{M} \mathrm{HCl}$ et $\mathrm{pH}<1$ avec $\mathrm{HNO}_{3} 65 \%$. Ces auteurs montrent que la désorption des métaux est plus forte à $\mathrm{pH} 3$ qu'à $\mathrm{pH} 6$, d'où une biodisponibilité plus grande pour les Poissons. La désorption est inférieure à $12 \%$ à pH 6 et inférieure à $35 \%$ à $\mathrm{pH} 3$, à l'exception du $\mathrm{Mn}$ et du $\mathrm{Cd}$ où la désorption est plus importante.

\subsection{Simulations de la digestion d'un Mollusque filtreur}

Comme dans le cas des actions de la salinité et du $\mathrm{pH}$, les attaques enzymatiques ont entraîné des désorptions plus importantes pour les prélèvements printaniers (crème de vase et sédiment de surface) que pour ceux de l'été (figs. 3 et 4).

$\mathrm{Au}$ printemps, les enzymes désorbent de façon significative le cadmium, notamment la pepsine (respectivement 54 et $38 \%$ pour le sédiment de fond et de surface), puis la lysosyme et la phosphatase acide (fig. 3).

En été, le cadmium est également le métal le plus facilement désorbé par la pepsine (surface et fond), la phosphatase acide, la peptidase et l'o-amylase. Le cuivre est assez bien désorbé par toutes les enzymes à l'exclusion de la peptidase, de la phosphatase alcaline et de la lysosyme (fig. 4).
Les grandes différences que nous observons dans nos estimations de la mobilité et de la biodisponibilité des éléments métalliques entre les échantillons de la crème de vase du printemps et de l'été peuvent s'expliquer par deux processus :

- soit par une variation du mode de liaison du métal selon le taux de matière organique présente dans l'échantillon. Dans nos deux échantillons la détermination de la concentration en matière organique par la méthode de la perte au feu donne des résultats très voisins : $8 \pm 2 \%$ au printemps et $11,7 \pm 0,3 \%$ en été;

- soit par le fait que l'échantillon prélevé en été était en présence d'une salinité plus élevée $(16,32 \%)$ qu'au printemps $(0,38 \%$ ) (Cf. tableau I). II est probable qu'en fonction de la salinité initiale, le mode de liaison métal-particule était différent dans les deux prélèvements, des liaisons plus faibles, donc plus aisément détruites, existant lorsque la compétition ionique pour les sites de fixation était minimale.

\section{CONCLUSIONS}

Expérimentalement, nous avons montré que la crème de vase prélevée dans l'estuaire de la Loire était susceptible de libérer une fraction des métaux qui lui étaient associés sous l'effet d'une augmentation de salinité. Ce relargage était d'autant plus marqué que la salinité initiale était plus faible. Ainsi pour les prélèvements estivaux $(16 \%$ ) la mobilité n'est mise en 


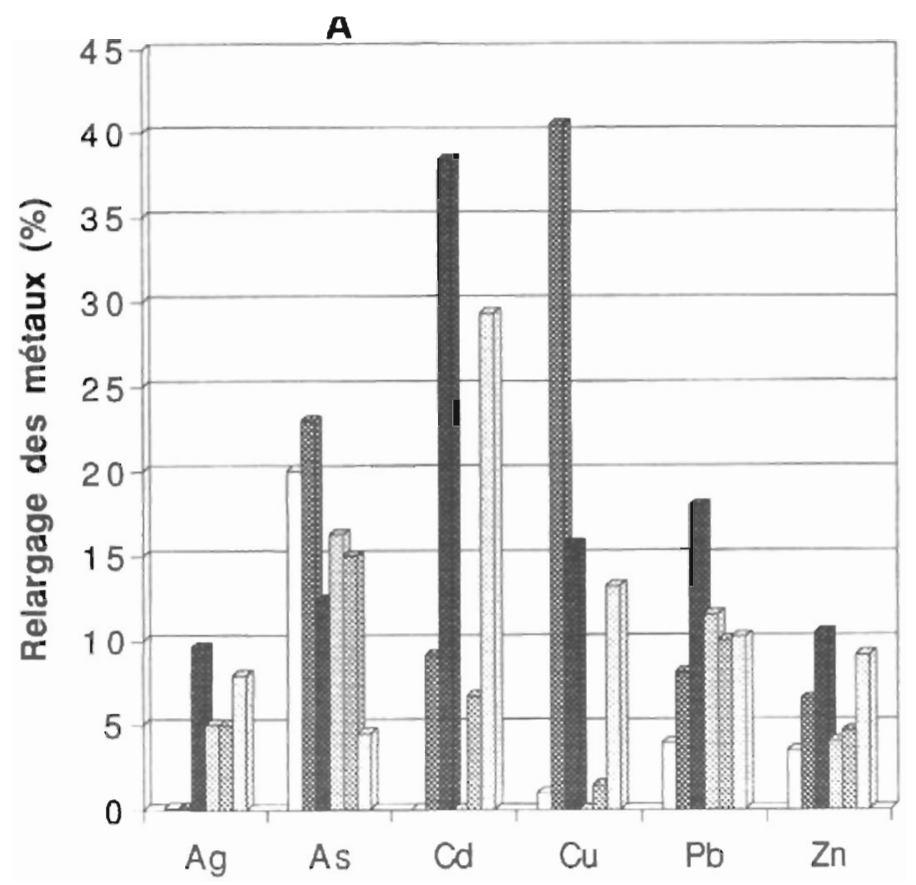

Lys. Amy. Peps. $\square$ Pept. $\quad$ Ph.Ac. $\square$ Ph.Alc.

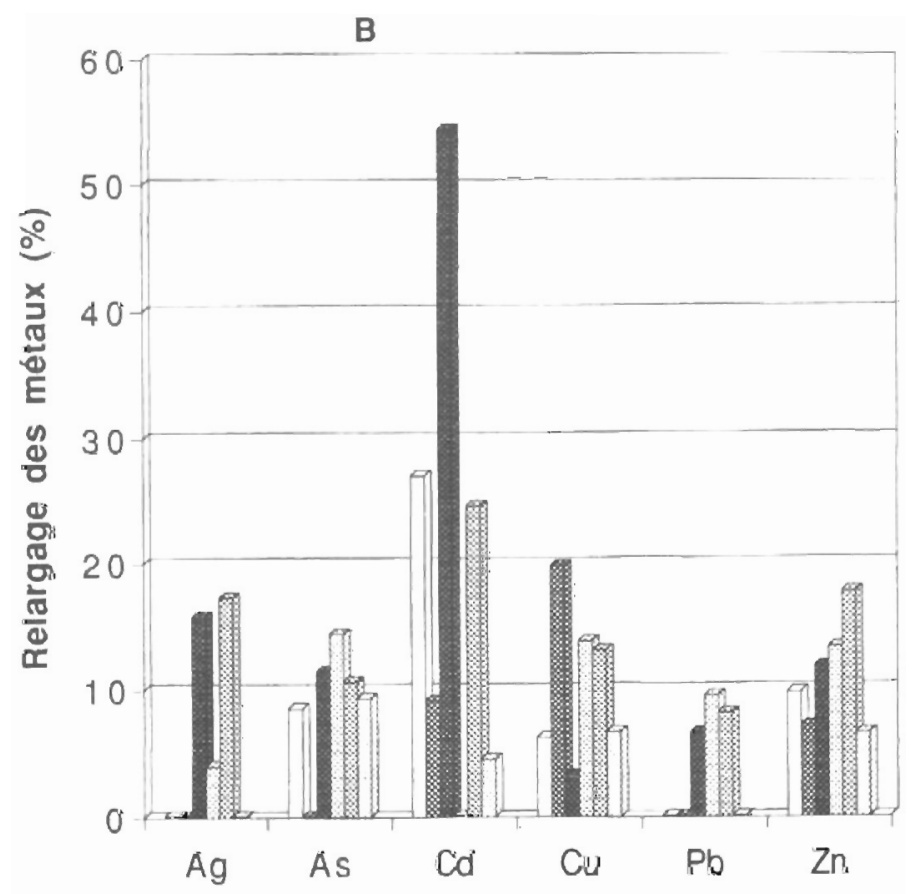

Fig. 3. - Pourcentages de désorption des éléments traces fixés sur les particules sédimentaires printanières du bouchon vaseux de l'estuaire de la Loire par diverses enzymes présentes dans le fube digestif des mollusques filtreurs (Lys. : lysozyme; Amy. : $\alpha$-amylase; Peps. : pepsine; Pept. : peptidase; Ph.Ac. : phosphatase acide; Ph.Alc. : phosphatase alcaline). A : particules de surface; B : particules du fond. 


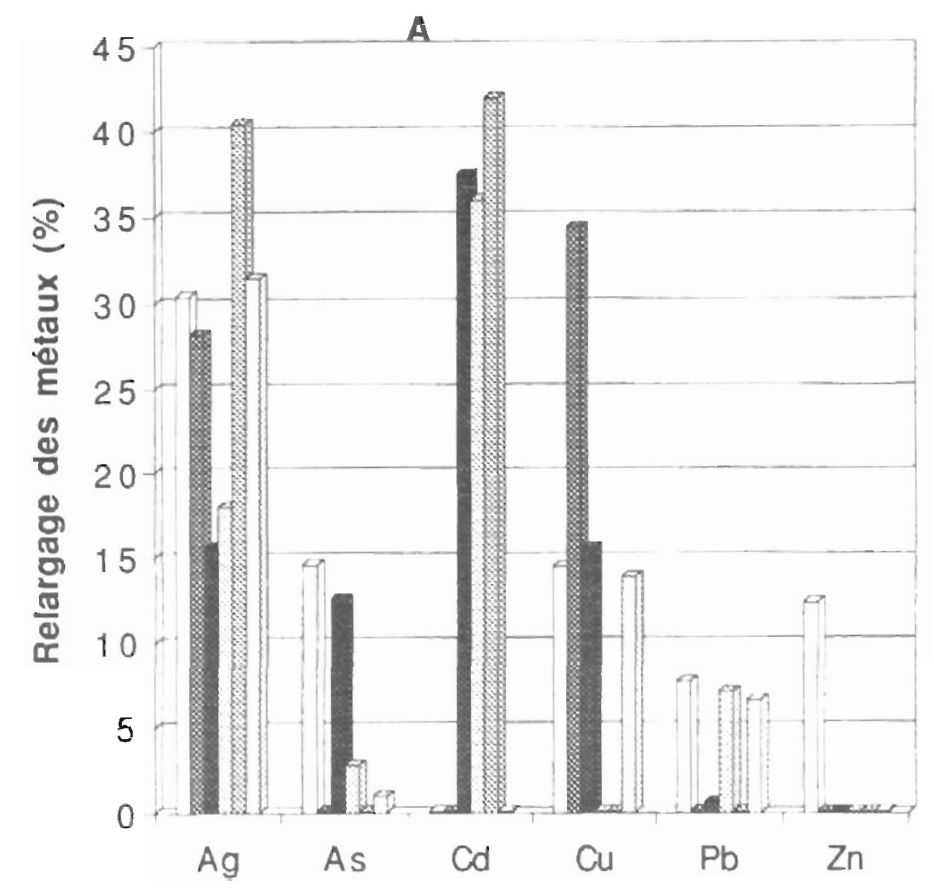

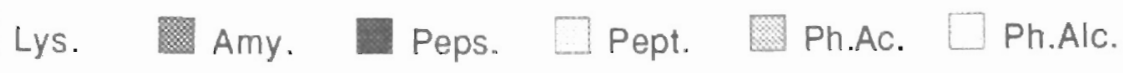

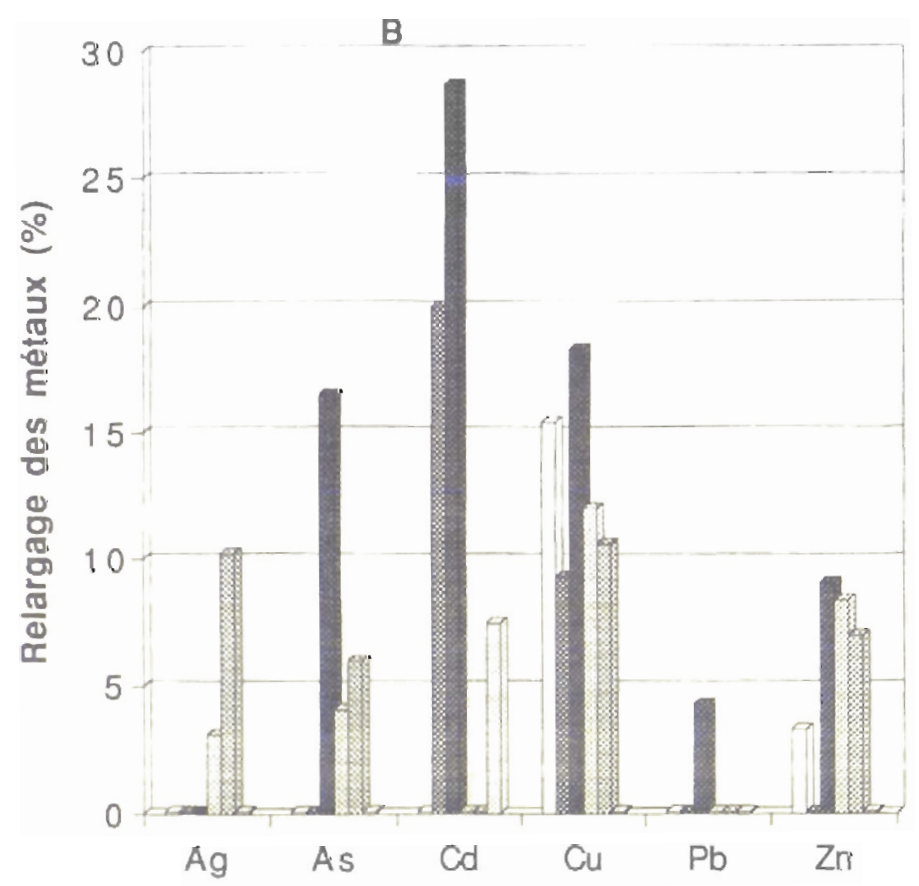

Fig. 4. - Pourcentages de désorption des éléments traces fixés sur les particules sédimentaires estivales du bouchon vaseux de l'estuaire de la Loire par diverses enzymes présentes dans le tube digestif des mollusques filtreurs (Lys. : Iysozyme; Amy. : $\alpha$-amylase; Peps. : pepsine ; Pept. : peptidase ; Ph.Ac. : phosphatase acide; Ph.Alc. : phosphatase alcaline). A : particules de surface; B: particules du fond. 
évidence que pour $\mathrm{As}$ et $\mathrm{Pb}$ alors que pour les prélèvements printaniers $(<1 \%)$ tous les métaux sont concernés ( $\mathrm{Ag}, \mathrm{As}, \mathrm{Cd}, \mathrm{Cu}, \mathrm{Pb}, \mathrm{Zn}$ ), les quantités libérées étant aussi plus importantes. Toutefois à ces variations de salinité peut se surimposer une évolution de la composition du bouchon vaseux (teneur et nature de la matière organique par exemple).

Nous ne disposons sur l'estuaire de la Loire que d'une seule étude de spéciation des métaux fixés aux particules (Nirel, 1987), mais seul le zinc est commun à nos deux études. De l'amont vers l'aval, une décroissance du zinc particulaire est observée mais elle n'est pas associée à une décroissance des fractions "labiles" et en particulier la part du zinc échangeable (fraction 1 de Tessier et al., 1979) est relativement plus importante. L'évolution du chrome en milieu estuarien ne serait pas contrôlée par des facteurs naturels mais dépendrait principalement d'une pollution locale: dans ces conditions les concentrations dans les MES augmentent globalement vers l'aval, l'enrichissement étant le plus marqué pour les fractions labiles. Cette observation peut-elle être généralisée aux autres éléments dont la présence dans l'estuaire est anthropique (As, $\mathrm{Pb}, \mathrm{Cu}, \ldots)$ ? En cas de réponse positive, ceci expliquerait la forte mobilité de ces éléments.

Soulignons toutefois que si l'on compare les teneurs en éléments métalliques des échantillons que nous avons prélevés (tab. 1B) avec le bruit de fond de la croûte terrestre (Försther et Wittman, 1979) ou avec les échantillons standard du NRC Canada, le degré de pollution reste modéré pour les trois métaux pré-cités et il n'est pas significatif pour $\mathrm{Cd}$ et $\mathrm{Zn}$. La portée de cette remarque doit être limitée en raison de la faible représentativité d'un échantillonnage dont l'objectif n'était pas d'établir le degré de pollution de l'estuaire ligérien.

Les attaques acides ou enzymatiques in vitro ont été menées en vue de simuler la désorption potentielle des métaux initialement liés aux particules, au cours de la digestion par les mollusques bivalves. La diminution du $\mathrm{pH}$ a un rôle dominant dans le relargage des métaux. Le métal donnant lieu aux phénomènes de désorption les plus marqués est le cadmium tandis que la mobilité de l'argent et de l'arsenic est plus modeste et que le plomb apparait comme le moins mobile des métaux testés.

Dans le cas des attaques enzymatiques, les enzymes ayant une action nette sur le relargage du cadmium sont différentes au printemps (pepsine, lysosyme et phosphatase acide) et en été (pepsine et $(\alpha$-amylase). Pour les prélèvements estivaux, le cuivre est désorbé par toutes les enzymes à l'exclusion de la phosphatase alcaline. Rappelons que Jones et Thomas (1988) avaient relevé des différences des formes labiles du cuivre selon l'origine des substances humiques notamment lors de blooms phytoplanctoniques. Ces résultats traduisent vraisemblablement des variations dans la nature des ligands présents dans la crème de vase au cours de l'année. 
II apparaît donc que les éléments liés aux particules ne sont pas définitivement mis à l'écart des cycles biogéochimiques puisque des processus de remise en solution pourraient intervenir au cours de la digestion.

\section{REMERCIEMENTS}

Nous tenons à remercier les membres de l'équipe du Port Autonome Nantes Saint-Nazaire et plus particulièrement Charles Casanova, pour l'aide qu'ils nous ont apporté dans l'échantillonnage. Cette étude a été en partie soutenue financièrement par le Syndicat Mixte d'Assainissement de la Rive Nord de la Loire (Nantes, France).

\section{BIBLIOGRAPHIE}

Amiard J.C., Pineau A., Boiteau H.L., Metayer C. \& Amiard-Triquet C., 1987. Application de la spectrométrie d'absorption atomique Zeeman aux dosages de huit éléments traces ( $\mathrm{Ag}, \mathrm{Cd}$, $\mathrm{Cr}, \mathrm{Cu}, \mathrm{Mn}, \mathrm{Ni}, \mathrm{Pb}$ et $\mathrm{Se}$ ) dans des matrices biologiques solides. Wat. Res., 21 (6) : 693-697.

Boniforti R., Bacciola D., Niccolai I. \& Ruggiero R., 1988. Selective extraction as an estimate of bioavailability of As, Cd, $\mathrm{Co}, \mathrm{Cr}, \mathrm{Cu}, \mathrm{Fe}, \mathrm{Mn}, \mathrm{Ni}, \mathrm{Pb}$ and $\mathrm{Zn}$ in marine sediments collected from the Central Adriatic sea. Environ. Technol. Letters, 9, 117-126.

Boucaud-Camou E., Lebesnerais C., Lubet P. \& Lihrmann I., 1983. Dynamique et enzymologie de la digestion chez I'huitre Crassostrea gigas (Thunberg). Bases biologiques de l'Aquaculture, Montpellier, IFREMER ed., Actes do Colloques, $n^{\circ} 1$ : 75-96.

Boust D., 1981. Les métaux-traces dans l'estuaire de la Seine et ses abords.
Thèse $3^{e}$ Cycle, Université de Caen, Rapport CEA -R-5104.

Bryan G.W., 1984. Pollution due to heavy metals and their compounds. In: Marine Ecology, O. Kinne, ed., 5, John Wiley \& Sons LTD, New-York, 12891431.

C.S.E.E.L. 1984. Rapport Final du Comité Scientifique pour l'Environnement de l'Estuaire de la Loire. CNEXO, Rapports Scientifiques et Techniques $n^{\circ} 55,158 \mathrm{p}$.

Campbell P.G.C., Lewis A.G., Chapman P.M., Crowder A.A., Fletcher W.K., Imber B., Luoma S.N., Stokes P.M. \& Winfrey M., 1988. Biologically available metals in sediments. Public. NRCC, Ottawa (Canada) Pub. No NRCC 27694, 298 p.

Deslous-Paoli J.M., 1987. Feeding and digestion with Bivalves. In : Nutrition in marine aquaculture, A. Bruno ed., Lisbon : 145-198.

El Sayed M., 1988. Contribution à l'étude du comportement géochimique de quelques métaux traces (cuivre, fer, manganèse et zinc) et de la matière organique dans le milieu estuarien. Cas de l'estuaire de la Loire et de la Rade de Brest. Thèse de Doctorat, Université de Bretagne Occidentale.

Etcheber H., 1979. Répartition et comportement du $\mathrm{Zn}, \mathrm{Pb}$, $\mathrm{Cu}$ et $\mathrm{Ni}$ dans l'estuaire de la Gironde. Bull. Inst. Géol. Bassin d'Aquitaine, Bordeaux, 25, 121-147.

Förstner U., 1990. Environmental factors controlling the uptake and releases of organic and inorganic pollutant burden. Proc. Intern. Seminar on the Environmental Aspects of Dredging Activities, Min. de l'Environ., ed. : 33 47.

Jones G.B. \& Thomas F.G., 1988. Effect of Terrestrial and Marine Humics on Copper Speciation in an Estuary in the Great Barrier Reef Lagoon. Aust. J. Mar. Freshwater Res., 39, 19-31. 
Jouanneau J.-M., Etcheber H. \& Latouche C., 1983. Imporishment and decrease of metallic elements associated with suspended matter in the Gironde estuary. In : Trace Metals in Sea Water. Proceeding of a NATO Advanced Research Institute, Erice 3/30-4/30/81. Wong et al., eds, Plenum Press, N.Y., 245-263.

Latouche C., Bertrand P., Etcheber H. \& Jouanneau J.-M., 1982. Comportement des oligo-éléments métalliques dans les milieux de transition eaux douces/eaux salées, cas du zinc dans l'estuaire de la Gironde. Mém. Soc. géol. Fr., N.S., 144, 155-160.

Lebesnerais C., 1985. Etude expérimentale de la digestion chez l'huître japonaise Crassostrea gigas (Thunberg). Thèse de doctorat, Université de Caen.

Luoma S.N., 1983. Bioavailability of trace metals to aquatic organisms. A Review. Sci. Total Environ., 28, 1-22.

Maest A.S., Crerar D.A., Stallard R.F. \& Ryan J.N., 1990. Metal and nutrient behavior in the Raritan estuary, New
Jersey, U.S.A. : The effect of multiple freshwater and industrial waste inputs. Chemistry Geology, 81 : 133-149.

Nirel P., 1987. Evolution de la distribution particulaire des éléments chimiques en milieu estuarien. Thèse de Doctorat, Université de Paris VII.

Seyler P., 1985. Formes chimiques et comportement de l'arsenic en milieu estuarien. Thèse de Doctorat, Université de Paris 6.

Tessier A., Campbell P.G.C. \& Bisson M., 1979. Sequential extraction procedure for the speciation of particulate trace metals. Anal. Chem., 51, 844-851.

Tessier A., Campbell P.G.C., Auclair J.C. \& Bisson M., 1984. Relationships between the partitioning of trace metals in sediments and their accumulation in the tissues of the freshwater mollusc Elliptio complanata in a mining area. Can. J. Fish. Aquat. Sci., 41, 14631472.

Yeats P.A. \& Loring D.H., 1991. Dissolved and particulate metal distributions in the St Lawrence estuary. Can. J. Earth Sci., 28 (5), 729-742. 